\title{
Politique
}

\section{Facteurs explicatifs des fluctuations de la popularité du président Reagan}

\section{Richard Nadeau et Guy Lachapelle}

Numéro 16, automne 1989

Les États-Unis

URI : https://id.erudit.org/iderudit/040634ar

DOI : https://doi.org/10.7202/040634ar

Aller au sommaire du numéro

Éditeur(s)

Société québécoise de science politique

ISSN

0711-608X (imprimé)

1918-6584 (numérique)

Découvrir la revue

Citer cet article

Nadeau, R. \& Lachapelle, G. (1989). Facteurs explicatifs des fluctuations de la popularité du président Reagan. Politique, (16), 35-58.

https://doi.org/10.7202/040634ar d'utilisation que vous pouvez consulter en ligne.

https://apropos.erudit.org/fr/usagers/politique-dutilisation/ 


\title{
Facteurs explicatifs des fluctuations de la popularité du président Reagan
}

\author{
Richard Nadeau \\ Université de Montréal \\ et \\ Guy Lachapelle \\ Université Concordia
}

John Mueller (1970, 1973), le pionnier des études sur la popularité présidentielle américaine, a dit un jour, après avoir constaté le déclin de la popularité de plusieurs présidents en cours de mandat, que les deux seuls moyens permettant au chef de l'exécutif américain de quitter la Maison-Blanche en demeurant populaire étaient, soit de démissionner le lendemain de son assermentation, soit de s'appeler Dwight David Eisenhower. Récemment, une certaine couverture médiatique a suggéré qu'il faudrait rajouter le nom de Ronald Reagan à cette très courte liste.

Le présent texte entend d'abord se pencher sur le bien-fondé de cette appréciation en examinant l'évolution de la popularité de ce dernier président. Ainsi, il nous sera possible de déterminer si la popularité du président Reagan a été faible ou élevée, stable ou fluctuante. Au-delà d'une analyse descriptive, c'est à une tentative 
d'explication des fondements de cette popularité que nous entendons nous consacrer.

La première partie de ce texte fait le bilan des études antérieures sur la popularité présidentielle en soulignant l'importance théorique de celle-ci et en en dégageant les principaux résultats. La deuxième partie répond à la question: Ronald Reagan a-t-il été un président populaire? Enfin la troisième partie s'interroge sur les fondements de cette popularité. Une brève conclusion présente un certain nombre de considérations théoriques et méthodologiques soulevées par notre étude.

\section{Les deux aspects de la popularité présidentielle}

La popularité présidentielle en tant que ressource politique

L'image que projette le président auprès de l'électorat a toujours représenté l'une des préoccupations majeures des responsables des administrations présidentielles. Richard Neustadt affirmait, au début des années soixante, que la question posée par la maison Gallup aux Américains, à savoir «s'ils approuvent ou désapprouvent la façon dont leur président s'acquitte de sa tâche», suscitait à Washington un intérêt constant. Neustadt allait même plus loin en suggérant que les membres du Congrès s'enquéraient régulièrement de l'état de l'opinion publique au sujet de la popularité présidentielle afin d'évaluer la vulnérabilité politique du chef de l'exécutif. Conséquemment, il en déduisait qu'un président populaire aurait plus de facilité à faire adopter son programme législatif ou à justifier une initiative diplomatique ou militaire (Neustadt, 1960). Les études récentes sur la popularité présidentielle ont repris à leur compte cette analyse:

"Neustadt observed more than twenty years ago that a president' standing in the approval polls greatly contributes to his public prestige, which in turn «is strategically important to his power» (Neustadt, 1960: 3). This insignt has been supported by 
subsequent quantitative work indicating that variations over time in congressional support for a president's legislative initiatives are systematically influenced by his Gallup approval ratings» (Hibbs, 1982a: 312-313).

Le maintien d'un haut niveau de popularité constituerait donc un objectif stratégique pour tout président (Ostrom et Simon, 1985). Les résultats les plus pertinents quant à cette question sont ceux de Rivers et Rose qui ont démontré qu'une hausse de un pour cent de la popularité présidentielle entraînait une augmentation du même ordre dans l'appui que le Congrès accordait au programme législatif du président (Rivers et Rose, 1985).

La popularité présidentielle présente donc deux aspects: 1 . en tant que ressource politique, elle délimite la marge de manoeuvre d'un président; 2 . en tant que manifestation de l'évaluation de la performance présidentielle, elle signale les préférences et les priorités des électeurs en matière de politiques étrangère et domestique. Ce deuxième aspect, intéressant en soi, prend une importance encore plus grande lorsque combiné avec le premier.

Les déterminants de la popularité présidentielle

La formulation la plus générale que l'on puisse établir afin de cerner les déterminants de la popularité serait la suivante:

Popularité = F (Économique, Politique).

La fonction de popularité présentée ci-dessus stipule simplement que la faveur dont jouit un gouvernement auprès de l'électorat est liée à l'évolution d'un certain nombre de variables économiques et politiques. Deux questions se posent immédiatement: quelles sont ces variables? Comment celles-ci sont-elles liées à la variable popularité?

Les réponses qui ont été données à ces questions ont établi que les principaux déterminants de la popularité seraient (Nadeau, 1988): 1. la prospérité économique (faible taux de chômage, rela- 
tive stabilité des prix, croissance économique soutenue); 2. les coûts d'une implication militaire prolongée (guerres de Corée et du Vietnam); 3. le ralliement en situation de crise internationale (crise des missiles à Cuba, ambassade américaine en Iran); 4. l'usure du pouvoir (cycle de popularité: période de lune de miel suivie d'une érosion de l'appui populaire); 5 . l'intégrité du président (affaires du Watergate, de l'Irangate); et 6 . la personnalité des acteurs politiques (Eisenhower, Kennedy). Les facteurs essentiels de la popularité présidentielle résideraient dans la prospérité, la paix et l'intégrité de l'administration au pouvoir (Kernell, 1978; Mackuen, 1983). L'étude de nos précédents déterminants a été regroupée selon trois rubriques: le cycle de la popularité, les variables économiques et les facteurs domestiques et internationaux et fait l'objet de quelques commentaires.

\section{Le cycle de la popularité}

L'hypothèse de l'existence d'un cycle de la popularité, c'està-dire d'une période de «lune de miel», suivie d'une période d'érosion de la popularité et d'une période de rétablissement de la popularité en fin de mandat, a d'abord été suggérée par Mueller (1970, 1973). Celui-ci en a proposé un traitement empirique et une justification théorique, reprenant l'argument de la coalition des minorités, établi par Anthony Downs (1957). Par la suite, James A. Stimson (1976a, 1976b) a enrichi cette thèse en suggérant l'existence d'un processus de gonflement irréaliste des attentes vis-à-vis un candidat en période électorale. Le contraste entre le niveau des attentes et l'action présidentielle serait donc à l'origine d'une dynamique d'érosion de la popularité dont Stimson fait, en dernière analyse, le facteur explicatif central, sinon unique, de la popularité présidentielle.

L'idée d'un cycle de popularité a toutefois été remise en question pour des raisons méthodologique et théorique. On a critiqué sévèrement cette variable, notamment sa faiblesse au plan théorique (Kernell, 1978; Presser et Converse, 1976; Sigelman et Smith, 1983, 1985). Hibbs en proposa une reformulation qui se 
heurta également à la critique (Hibbs, 1982a, 1982b, 1982c; Keech, 1982: Norpoth, 1984). Finalement, après plus de vingt ans de débats, il ne subsiste de cette hypothèse que l'observation de l'existence d'une période de «lune de miel», variable qui a su résister aux pratiques économétriques les plus sophistiquées (Norpoth, 1984; Lanoue, 1987).

Les variables économiques

Les débats ont porté à la fois sur le choix des variables (Kernell, 1978; Hibbs, 1982a, 1982b, 1982c; Lanoue, 1987), sur leur utilisation (Mueller, 1979, 1973; Kenski, 1977; Hibbs, 1982a, 1982b, 1982c) et sur les pratiques économétriques (Norpoth, 1984). D'autres controverses ont également eu pour objet l'information pertinente à la prise de décision électorale (Kiewett, 1982) et la rationalité sous-jacente au processus d'évaluation de la politique économique (Chappel et Keech, 1985).

À partir des résultats des études sur l'impact des variables économiques, trois tendances se dégagent: 1. des trois indicateurs macroéconomiques les plus utilisés (chômage, inflation, revenu personnel disponible), l'inflation ressort comme le facteur déterminant de la popularité présidentielle pour la période 1952-1984. Le revenu se classe au deuxième rang, suivi du chômage. 2. Les meilleurs résultats ont été obtenus avec des structures courtes de délais (généralement un ou deux trimestres de décalage); 3. les variables économiques contribuent à expliquer près du tiers de la variance expliquée mais les résultats obtenus restent sujet à caution à cause de l'instabilité des relations observées (Beck, 1983; Whiteley, 1980, 1984).

\section{Les variables domestiques et internationales}

De manière générale, les variables relatives à la gestion présidentielle des enjeux internationaux semblent jouer un rôle beaucoup plus important que les préoccupations d'ordre domestique 
(Mueller, 1970, 1973; Kernell, 1978; Mackuen, 1983; Ostrom et Simon, 1985); cette constatation est surtout exacte dans les cas d'une implication militaire prolongée des États-Unis, comme lors des guerres de Corée et du Vietnam, et également dans les situations de tension internationale. Les variables domestiques, quant à elles, jouent un rôle nettement plus modeste comme facteur explicatif de la popularité présidentielle, exception faite de la variable intégrité, comme dans le cas de l'affaire du Watergate.

Il est toutefois curieux de constater que ces résultats n'ont pas amené les spécialistes à établir un lien entre les déterminants de la popularité présidentielle, et la thèse des deux présidences avancée par Wildavsky (1975), puis reprise par LeLoup et Shull (1979). S'il est vrai que le président américain dispose d'une marge de manoeuvre importante lorsqu'il s'agit de la politique étrangère et qu'il est menotté par le Congrès pour ce qui est de la politique intérieure, il serait logique de supposer que sa popularité est davantage liée à la gestion des questions internationales et cela, pour deux raisons: 1 . à cause du partage des responsabilités entre le Congrès et la Maison-Blanche, et 2. à cause de l'activité du président, plus manifeste et plus importante dans le champ d'activité où il a les coudées franches. Ces résultats présentent aussi des implications du point de vue de la gestion optimale de l'agenda politique par un président.

Les performances macroéconomique et diplomatique des présidents américains expliqueraient donc, pour une bonne part, les fluctuations de leur popularité et nous allons maintenant évaluer si cela s'avère juste dans le cas du président Reagan. Ce faisant, nous saurons si la hiérarchie des effets suggérée par les écrits - affaires étrangères, affaires domestiques, taux d'inflation, taux de chômage — s'est maintenue durant ses deux mandats. 


\section{Le président Reagan:}

le mythe d'un président populaire

À la suite de son élection en 1980, mais surtout au lendemain du raz-de-marée de 1984, le président Reagan semble avoir réussi à projeter l'image d'un président populaire qui a su s'attirer l'appui de toutes les couches de la société américaine. S'agit-il uniquement d'un phénomène médiatique? Au contraire, peut-on affirmer que le président Reagan a été l'un des présidents les plus populaires depuis Harry Truman? C'est à ces deux questions que nous tenterons de répondre en comparant la popularité du président Reagan à celle de ses prédécesseurs.

Le taux d'approbation de la performance présidentielle représente, depuis 1945, l'une des rares variables permettant de suivre à la trace les variations de l'appui populaire au président des États-Unis. Les premiers résultats concernant la popularité présidentielle remontent à Harry Truman, quelques mois après son accession à la présidence à la suite du décès de Franklin D. Roosevelt. Trois mois après son arrivée à la Maison-Blanche, le président Truman jouit d'une très grande popularité puisque $87 \%$ des Américains se disent satisfaits de sa performance. Toutefois, un an plus tard, en mars 1946, cet appui baisse à 50\% pour continuer de chuter et atteindre $32 \%$ l'année suivante. À la fin de sa présidence, la popularité de Truman se retrouve sous la barre des $30 \%$. Ces fluctuations (un écart de plus de 60 points entre le début et la fin d'un exercice présidentiel) sont les plus importantes qui aient été observées depuis que la maison Gallup recueille des informations sur le taux d'approbation de la performance présidentielle.

De telles fluctuations contrastent avec l'étonnante stabilité de la popularité de son successeur, Dwight Eisenhower, comme on peut l'observer au tableau 1. En effet, la capacité de ce dernier à maintenir un haut niveau d'appréciation de sa gestion présidentielle apparaît comme un cas d'espèce de la vie politique américaine (Mueller, 1970, 1973; Stimson, 1976a, 1976b; Kernell, 1978).

John Kennedy fut aussi un président populaire. Au cours de sa première année, il reçut l'appui de $75.9 \%$ des Américains. Ce taux d'approbation atteignit même le sommet de $79 \%$ en septem- 
bre 1961 et en janvier 1962. Kennedy est en fait le président américain qui a su maintenir, au cours de la première année de son exercice, la plus haute cote de popularite. Même si sa popularité était en déclin au moment de son assassinat, en novembre 1963 , ayant reculé de vingt points en moins de deux ans, il fut, malgré tout, l'une des figures politiques les plus populaires qu'aient connues les États-Unis.

Son successeur, Lyndon Johnson, a connu, au cours de sa première année, une popularité assez semblable à celle de Kennedy, se situant à $75.1 \%$ pour atteindre le sommet de $78 \%$ en mai 1964. Par la suite, sa popularité ne cessa de décroître pour se retrouver, quarante-huit mois après son arrivée à la MaisonBlanche, à $42 \%$, un recul de plus de 36 points par rapport à mai 1964. Malgré tout, Lyndon Johnson semble avoir été l'un des derniers de cette lignée de présidents qui pouvaient jouir, dès leur première année au pouvoir, d'un appui avoisinant les $70 \%$.

Avec l'élection de Richard Nixon en 1968, une nouvelle période débute puisque les électeurs américains semblent moins enclins à donner un appui massif à leur nouveau président. Ainsi, la popularité du président Nixon au cours de sa première année se retrouve nettement en deça de celle de ses prédécesseurs, se situant en moyenne à $61.2 \%$. En fait, seuls Richard Nixon et Ronald Reagan ne réussiront pas à dépasser la barre des $69 \%$ au cours de leur première année de présidence. Par la suite, la popularité du président Nixon atteindra ce niveau (69\%) lors de la signature de l'entente mettant fin au conflit américano-vietnamien. Mais les révélations concernant l'affaire du Watergate en 1973 mineront cet appui qui diminuera de plus de 30 points. Son successeur, Gérald Ford, jouira, quant à lui, d'une approbation plutôt mitigée au cours de sa première année, recevant l'appui de seulement $46 \%$ des Américains, le plus faible taux d'approbation obtenu par un président après douze mois au pouvoir. Bien que sa popularité ait atteint les $71 \%$ au moment de son investiture (avant le pardon présidentiel accordé à Richard Nixon), il fut le président le moins populaire au cours de sa première année d'exercice. 
Tableau 1: La popularité présidentielle au cours de la première année et durant le premier mandat $(\text { en } \%)^{1}$

\begin{tabular}{|c|c|c|c|c|c|c|c|c|}
\hline & $\begin{array}{c}\text { Truman } \\
(4 / 45)\end{array}$ & $\begin{array}{c}\text { Elsenhower } \\
\text { (1/53) }\end{array}$ & $\begin{array}{c}\text { Kennedy } \\
\text { (1/61) }\end{array}$ & $\begin{array}{c}\text { Jobnson } \\
\text { (1/63) }\end{array}$ & $\begin{array}{l}\text { Nixan } \\
(1 / 69)\end{array}$ & $\begin{array}{l}\text { Ford } \\
(8 / 74)\end{array}$ & $\begin{array}{l}\text { Carter } \\
(1 / 77)\end{array}$ & $\begin{array}{r}\text { Reagan } \\
(1 / 81)\end{array}$ \\
\hline \multicolumn{9}{|l|}{ a. Premixte annce } \\
\hline Janvier & - & - & - & - & 59 & 71 & - & - \\
\hline Aprts un mois & - & 68 & 72 & 74 & 60 & 50 & 71 & 55 \\
\hline Apres deux mois & - & 59 & 73 & 77 & 66 & 54 & 72 & 60 \\
\hline Apres trois mois & 87 & 74 & 78 & 74 & 60 & 48 & 63 & 67 \\
\hline Apres quatre mois & - & 74 & 75 & 78 & 62 & 42 & 64 & 68 \\
\hline Apuess cinq mois & 一 & - & 72 & 75 & 63 & 37 & 63 & 58 \\
\hline Apriss six mois & - & 71 & - & 74 & 63 & 38 & 67 & 56 \\
\hline Aprits sept mois & 75 & - & 76 & 74 & 62 & 37 & 66 & 60 \\
\hline Apres huit mois & - & 75 & 79 & 一 & 58 & 39 & 54 & 52 \\
\hline Apres neuf mois & - & 65 & 77 & - & 56 & 40 & 51 & 56 \\
\hline Apres dix mois & 63 & - & 79 & - & 67 & 52 & 56 & 54 \\
\hline Aprits onze mois & $=$ & 60 & 78 & $=$ & 59 & - & 57 & 49 \\
\hline Apress douze mois & 50 & 68 & 79 & 70 & 63 & 46 & 52 & 47 \\
\hline Moyenne & 68,8 & 68,2 & 75,9 & 75,1 & 61,2 & 46,2 & 62,2 & 57,7 \\
\hline Sommet & 87 & 75 & 79 & 78 & 67 & 71 & 72 & 68 \\
\hline \multicolumn{9}{|l|}{ b. Premier mandat } \\
\hline Apres 13 mois & - & 71 & 78 & 71 & 64 & 45 & 50 & 47 \\
\hline Après 24 mois & 57 & 70 & 74 & 62 & 57 & 一 & 43 & 35 \\
\hline Apress 36 mois & 36 & 77 & 58 & 49 & 50 & 一 & 58 & 54 \\
\hline Apres 48 mois & - & 75 & - & 42 & 62 & - & 29 & 58 \\
\hline
\end{tabular}

(1) Les taux d'approbation de ce tableau sont exprimés en pourcentage du nombre de répondants indiscrets.

Source: Public Opinion, février-mars 1984, p. 34. 
Jimmy Carter fait aussi partie de ce groupe de présidents ayant eu de la difficulté à maintenir un niveau de popularité élevé au cours de leur première année à la Maison-Blanche. Durant cette période, sa popularité atteindra le sommet de $72 \%$, pour se situer en moyenne à $62.2 \%$, un score supérieur à celui de Gérald Ford mais comparable à celui de Richard Nixon. Les fluctuations de la popularité de ce président seront généralement descendantes, sauf au cours de la prise des otages à l'ambassade américaine de Téhéran, et très accentuées. Le score de $58 \%$ enregistré par Jimmy Carter après trente-six mois peut surprendre. On doit ici rappeler les circonstances particulières de la fin de l'année 1979 alors que les Américains, sous le choc de la prise d'otages à l'ambassade américaine en Iran, s'étaient massés derrière leur président, contribuant ainsi à faire augmenter la popularité de ce dernier de dix-huit points en deux mois. Cette popularité augmentera encore un peu après l'invasion soviétique en Afghanistan. L'effet de ces deux événements s'est cependant résorbé en moins de deux trimestres.

Quant à Ronald Reagan, sa popularité moyenne s'est établie à $57.7 \%$, au cours de sa première année à la Maison-Blanche, un score moyen nettement en deçà de celui de tous ses prédécesseurs, à l'exception de Gérald Ford (46.2\%). De plus, avec un taux d'approbation de $47 \%$, douze mois après son élection, le président Reagan se situe sous la barre des $50 \%$, ce qui en fait, encore après Gérald Ford, le président le moins populaire de l'après-guerre lors d'une première année à la présidence des ÉtatsUnis. Vingt-quatre mois après son élection, Ronald Reagan ne recevait plus que 35\% de l'appui populaire, une des plus faibles performances au cours de la période étudiée. Par la suite, la popularité du président Reagan se redressera, atteignant $60 \%$ au moment de sa rélection.

En somme, il apparaît que la perception selon laquelle Ronald Reagan aurait été un président populaire au cours de son premier mandat semble relever davantage du mythe que de la réalité puisque sa popularité se situe, de manière générale, en deçà de celle de ses prédécesseurs. Finalement, sa popularité au début de son second mandat, en janvier 1985 , se retrouve à $62 \%$, nettement sous celle des Truman (69\%), Eisenhower (73\%) et Johnson 
(71\%) mais au-dessus de celle de Richard Nixon (51\%). La thèse du président populaire, dans le cas de Ronald Reagan, n'est donc pas démontrée.

\section{L'analyse empirique:}

les déterminants de la popularité du président Reagan

\section{Une vue d'ensemble}

Le tableau 2 fournit une vue d'ensemble du contexte dans lequel se sont déroulés les vingt-sept premiers trimestres de la présidence de Ronald Reagan et suggère une série de pistes relativement aux déterminants de la popularité de ce dernier. On constate d'abord que le taux d'approbation de la gestion présidentielle de Ronald Reagan a été d'environ 60\% durant les sept premières années de sa présidence. Ce taux s'avère nettement supérieur au taux d'approbation de sa politique économique $(+9.7$ points), de sa politique étrangère $(+7.4)$ et de sa politique en Amérique centrale (+21.5). Il est cependant légèrement inférieur au taux d'approbation concernant les relations avec l'URSS (-1.2). Une première hiérarchisation des appuis apparaît donc: IURSS, la performance d'ensemble, la politique étrangère, la politique économique et la politique touchant l'Amérique centrale.

Cette hiérarchisation ne s'est que partiellement maintenue au cours des mandats à l'étude. Une constante demeure cependant: les taux d'approbation concernant les relations avec l'URSS ont toujours dominé ceux relatifs aux politiques économique et étrangère, le taux le plus élevé restant celui touchant les rapports avec Moscou et le plus faible, et de loin, se rapportant à la politique présidentielle en Amérique centrale. Cependant, les rangs relatifs des politiques étrangères et économique se sont modifiés d'un mandat à l'autre, suggérant une première observation à propos de l'appui présidentiel, à savoir que la popularité de Ronald Reagan aurait surtout reposé sur la politique étrangère durant son premier mandat et sur la politique économique durant le second. 
Tableau 2: Taux d'approbation général et spécifiques, taux de chómage et d'inflation, pourcentages de répondants identifiant les problèmes économiques ou internationaux comme les plus importants auxquels les États-Unis étaient confrontés durant la présidence de Ronald Reagan 1

\begin{tabular}{|c|c|c|c|c|c|c|c|c|c|}
\hline \multirow{2}{*}{$\begin{array}{l}\text { Taux d'approbation, de } \\
\text { chómage, d'inflation et } \\
\text { d'identification du pro- } \\
\text { blame be plus important }{ }^{2}\end{array}$} & \multirow[b]{2}{*}{ Global } & \multicolumn{4}{|c|}{ Taux d'approbation (en \% des indiscrets) } & \multicolumn{2}{|c|}{ Twax } & \multicolumn{2}{|c|}{ Probleme le + importent } \\
\hline & & $\begin{array}{l}\text { Polit. } \\
\text { Econo- } \\
\text { mique }\end{array}$ & $\begin{array}{l}\text { Polit. } \\
\text { Gtrun- } \\
\text { gert }\end{array}$ & URSS & $\begin{array}{l}\text { Amérique } \\
\text { centrale }\end{array}$ & chome & $\begin{array}{c}\mathbf{d}^{\prime} \\
\text { inflation. }\end{array}$ & Economique & International \\
\hline $\begin{array}{l}\text { Deux mandats (moyeme) } \\
\text { Premier mandat (moyenne) } \\
\text { Deuxieme mendat (moyenne) }\end{array}$ & $\begin{array}{l}59,5 \\
56,7 \\
63,4\end{array}$ & $\begin{array}{l}49,8 \\
47,9 \\
52,6\end{array}$ & $\begin{array}{l}52,1 \\
53,2 \\
50,4\end{array}$ & $\begin{array}{l}60,7 \\
58,5 \\
63,8\end{array}$ & $\begin{array}{l}48,0 \\
38,6 \\
37,0\end{array}$ & $\begin{array}{l}7,9 \\
8,6 \\
6,9\end{array}$ & $\begin{array}{l}3,9 \\
4,5 \\
3,1\end{array}$ & $\begin{array}{l}57,1 \\
65,4 \\
45,1\end{array}$ & $\begin{array}{l}16,9 \\
14,3 \\
20,7\end{array}$ \\
\hline $\begin{array}{l}\text { Minimum: deux mandats } \\
\text { Maximum: ceux mandats }\end{array}$ & $\begin{array}{l}42,0 \\
75,4\end{array}$ & $\begin{array}{l}31,1 \\
63,6\end{array}$ & $\begin{array}{l}36,7 \\
70.3\end{array}$ & $\begin{array}{l}50,6 \\
75,3\end{array}$ & $\begin{array}{l}26,4 \\
50,3\end{array}$ & $\begin{array}{r}6,0 \\
10,7\end{array}$ & $\begin{array}{r}1.7 \\
10.2\end{array}$ & $\begin{array}{l}40,5 \\
80,2\end{array}$ & $\begin{array}{r}6,5 \\
25,9\end{array}$ \\
\hline $\begin{array}{l}\text { Minimum: premier mandat } \\
\text { Maximum: premier mandat }\end{array}$ & $\begin{array}{l}42,0 \\
75,4\end{array}$ & $\begin{array}{l}31,1 \\
63,6\end{array}$ & $\begin{array}{r}40,2 \\
70.3\end{array}$ & $\begin{array}{l}50,6 \\
75,3\end{array}$ & $\begin{array}{l}30,5 \\
50,3\end{array}$ & $\begin{array}{r}7,3 \\
10,7\end{array}$ & $\begin{array}{r}1,7 \\
10,2\end{array}$ & $\begin{array}{l}50,2 \\
80,2\end{array}$ & $\begin{array}{r}6,5 \\
24,2\end{array}$ \\
\hline $\begin{array}{l}\text { Minimum: deuxizme mandat } \\
\text { Mzximum: deluxidmo mandat }\end{array}$ & $\begin{array}{l}52,7 \\
70,5\end{array}$ & $\begin{array}{l}46,8 \\
58,2\end{array}$ & $\begin{array}{l}36,7 \\
59,8\end{array}$ & $\begin{array}{l}57,6 \\
74,7\end{array}$ & $\begin{array}{r}26,4 \\
45,4\end{array}$ & $\begin{array}{l}6,0 \\
7,3\end{array}$ & $\begin{array}{l}1,8 \\
4,8\end{array}$ & $\begin{array}{l}40,5 \\
50,4\end{array}$ & $\begin{array}{l}18,3 \\
25,9\end{array}$ \\
\hline $\begin{array}{l}\text { Ecart-type: deux mandats } \\
\text { Ecart-type: premier mandat } \\
\text { Ecart-type: deuxieme mandat }\end{array}$ & $\begin{array}{l}1,8 \\
2,4 \\
2,2\end{array}$ & $\begin{array}{l}1,6 \\
2,6 \\
1,1\end{array}$ & $\begin{array}{l}1,8 \\
2,3 \\
3,0\end{array}$ & $\begin{array}{l}1,4 \\
1,9 \\
1,6\end{array}$ & $\begin{array}{l}1,2 \\
1,4 \\
2,1\end{array}$ & $\begin{array}{l}0,25 \\
0,31 \\
0,13\end{array}$ & $\begin{array}{l}0,40 \\
0,60 \\
0,32\end{array}$ & $\begin{array}{l}2,5 \\
2,7 \\
1,1\end{array}$ & $\begin{array}{l}1,2 \\
1,8 \\
0,7\end{array}$ \\
\hline
\end{tabular}

(1) Les donnes renvoient aux questions suivantes: pour be taux d'approbation global: «Do you approve of the way Ronald Reagan is handling his job as President?»; et pour les taux d'approbetion specifiques: «Do you approve of the way Ronald Reagan is handling that problem: Foreign Policy Relations with the Soviet Union? The situation in Central America? Economic conditions in this country?s. L'information sur le probleme lo plus important renvoie aux réponses obtenus i la question: *What is the most important problem facing the country today?*.

(2) Le nombre d'observations pour les taux d'approbation et les variables tconomiques est de vingt-eept (27). Dans les ces des taux d'approbation, quelques interpolations ont Été effectués pour ussurer la continuité des séries chronologiques. Les donnbes sur lo problème le plus important sont nu nombre de quatorze (14). Le fichier de donnses brutes des auteurs est accessible sur demande.

Sources: The Gallup Report, 1981-1987, numéros divers; U.S. Department of Commerce, Business Conditions Digest (Bureau of Economic Analysis, février 1988), Public Opinion Quarterly, divers numéros. 
Cette première hypothèse semble confirmée par l'observation des taux de chômage et d'inflation, systématiquement plus bas durant le second mandat. Cette amélioration de la conjoncture économique a entraîné une modification de la composition de l'agenda politique ainsi que l'atteste la chute très importante, durant le second mandat (-20.3 points), du pourcentage d'Américains identifiant l'un ou l'autre de ces problèmes économiques comme étant le plus important auquel était confronté leur pays. Ces données, qui nous permettent de voir des Américains moins préoccupés par les problèmes économiques et généralement satisfaits de la gestion macroéconomique du président, suggèrent que l'approbation de celle-ci pourrait avoir été un point d'appui important pour Ronald Reagan durant son deuxième mandat ainsi que semblent l'attester le niveau d'approbation (52.6 pour cent; gain de près de cinq points par rapport au mandat précédent) mais surtout la stabilité de l'appui reçu pour sa politique économique (minimum de $46.8 \%$ et écart-type très faible).

Le fait que les pourcentages d'appuis aux politiques étrangère et centre-américaine aient été plus faibles durant le second mandat, et que les minimums d'appuis de ces politiques aient été enregistrés durant cette période, vont également dans le sens des observations précédentes. Le grand succès de la diplomatie reaganienne a été sa politique soviétique. Au creux de la récession de 1982 comme dans les pires moments du scandale de l'Irangate, les Américains ont toujours continué à appuyer massivement ce volet de sa politique. Si l'on garde à l'esprit l'importance de cet enjeu pour les Américains mais aussi, et plus encore, les attentes très précises qu'ils entretenaient relativement à la capacité de Ronald Reagan de traiter ce dossier (Sigelman et Smith, 1985), on doit conclure qu'il a bien manoeuvré, ce qui lui a valu des appuis importants.

Il apparaît donc que se sont les succès dans les politiques soviétique et économique de sa gestion présidentielle qui ont fourni à Ronald Reagan le capital d'appuis nécessaires pour se maintenir durant une crise aussi sérieuse que celle de l'Irangate. L'explication est moins imagée que celle d'un «président - teflon» mais sans doute plus conforme aux faits. 
Tableau 3: corrélations simples des taux d'approbation global et spécifiques, des taux de chômage et d'inflation 1

\begin{tabular}{|c|c|c|c|c|c|c|c|}
\hline & \multicolumn{5}{|c|}{ Taux d'approbation } & \multicolumn{2}{|c|}{ Taux } \\
\hline & Global & $\begin{array}{l}\text { politique } \\
\text { étrangère }\end{array}$ & $\begin{array}{l}\text { relations } \\
\text { avec l'URSS }\end{array}$ & $\begin{array}{c}\text { Amérique } \\
\text { centrale }\end{array}$ & $\begin{array}{l}\text { politique } \\
\text { économique }\end{array}$ & de chômage & d'inflation \\
\hline \multicolumn{8}{|l|}{ a) Deux mandats } \\
\hline Global & - & 0,75 & 0,78 & 0,66 & 0,91 & $-0,68$ & 0,33 \\
\hline Politique étrangère & - & - & 0,71 & 0,89 & - & - & - \\
\hline Politique économique & & - & - & - & - & $-0,76$ & 0,38 \\
\hline \multicolumn{8}{|l|}{ b) Premier mandat } \\
\hline Global & - & 0,83 & 0,76 & 0,76 & 0,96 & $-0,89$ & 0,80 \\
\hline Politique étrangère & - & - & 0.92 & 0.89 & - & - & - \\
\hline Politique économique & - & - & - & - & - & $-0,92$ & 0,65 \\
\hline \multicolumn{8}{|l|}{ c) Deuxième mandat } \\
\hline Global & - & 0.98 & 0,73 & 0,84 & 0,84 & 0,75 & $-0,74$ \\
\hline Politique étrangère & - & - & 0,71 & 0,89 & - & - & - \\
\hline Politique économique & - & - & - & - & - & 0,70 & $-0,74$ \\
\hline
\end{tabular}

(1) Seules les corrélations semblant renvoyer à un lien causal explicite sont présentées. 


\section{L'économétrie de la popularité}

Le tableau 3 présente une série de coefficients de corrélations simples entre les principales variables de notre étude. Deux résultats se dégagent de l'examen de ces coefficients: 1. la corrélation est plus élevée entre le taux d'appréciation global et relatif de la politique économique qu'entre le taux global et le taux concernant la politique étrangère $(0.91$ versus 0.75$) ; 2$. la caractérisation des deux mandats présidentiels sous l'angle des déterminants de la popularité, le premier ayant été principalement marqué par des préoccupations économiques (corrélation de 0.96 entre les taux d'approbation global et économique) et le second plus orienté vers des préoccupations en matière de politique étrangère (corrélation de 0.98 entre les taux d'approbation global et relatif à la politique extérieure).

Les autres résultats importants concernent les déterminants des taux d'appréciation spécifique. S'agissant du taux d'appréciation de la politique économique, le facteur dominant, et de loin, est le taux de chômage (corrélation de 0.76), la corrélation entre ce taux et l'inflation étant plus faible et de signe contraire à l'attente théorique, probablement à cause de la fonction identique jouée par le chômage et l'inflation. Ce lien étroit entre chômage et évaluation de la politique économique disparaît lors du second mandat au profit de l'inflation. Notre interprétation est la suivante: durant le second mandat, le chômage a peu varié et la politique étrangère a été dominante; la corrélation simple entre chômage et popularité est maintenant brouillée par l'importance prise par les événements internationaux dans l'évaluation de la gestion présidentielle. En fait, durant le second mandat, les questions économiques ont été quelque peu reléguées au second plan de sorte qu'au moment où le taux de chômage recommence à diminuer fin 1986, début 1987, et que la popularité du président Reagan connaît une chute importante, subissant les contrecoups de l'Irangate, les problèmes économiques n'ont plus l'acuité du premier mandat et une corrélation (fallacieuse) positive simple semble s'établir entre le taux de chômage et la popularité. 
Le taux d'approbation de la politique étrangère semble avoir été davantage lié au taux d'approbation de la politique centreaméricaine du président qu'à sa politique soviétique (coefficients de 0.89 et de 0.71). Le fait n'apparaît cependant qu'au second mandat, alors que l'appui à la politique soviétique de Ronald Reagan se maintient à un haut niveau alors que la politique étrangère en général, celle en Amérique centrale en particulier, est désavouée. C'est durant ce mandat que le caractère autonome de la question soviétique ressort le plus clairement. L'analyse de corrélation simple présente donc une image des déterminants de la popularité conforme aux résultats précédemment obtenus: la performance économique domine, suivie de la politique soviétique et de la politique étrangère en général, la prestation présidentielle en Amérique centrale étant décisive en ce qui concerne la politique étrangère.

Il reste maintenant à effectuer le test pour cette hiérarchisation: l'analyse de régression multiple. Les tableaux 4 et 5 résument notre démarche. Nous avons d'abord procédé à une analyse de régression simple (tableau 4) afin de voir les déterminants des taux d'approbation global et spécifique. En nous appuyant sur ces résultats, nous avons ensuite fait une analyse de régression multiple (tableau 5).

Les informations du tableau 4 ont été organisées afin de faire ressortir la hiérarchisation des déterminants de la popularité présidentielle. En examinant les pourcentages de variance expliquée, la hiérarchisation observée est la suivante: taux d'approbation de la politique économique, de la politique concernant les relations avec l'URSS, de la politique étrangère, taux de chômage, taux d'approbation de la politique centre-américaine et finalement, loin derrière, taux d'inflation. Par ailleurs, le taux de chômage domine encore très nettement le taux d'inflation en tant que déterminant de l'évaluation de la politique économique. Il ressort que le taux d'approbation de la politique présidentielle en Amérique centrale est davantage lié au taux d'approbation de la politique étrangère que ne l'est la politique soviétique. Ces résultats, conformes aux précédents, ont orienté notre analyse de régression multiple. 


\section{FLUCTUATIONS DE LA POPULARTTÉ DU PRESIDENT REAGAN 51}

\section{Tableau 4: Une analyse de régression simple des dé- terminants de la popularité du président Reagan1}

a) TAUX D'APPROBATION GLOBAL

\begin{tabular}{|c|c|c|c|c|c|}
\hline $\mathrm{TA}$ (GL) & $=$ & $\begin{array}{l}11.1 \\
(2.5)^{* * *}\end{array}$ & + & $\begin{array}{l}0.97 \text { TA(ECN }) \\
(11.3)^{* * * 3}\end{array}$ & $0.83^{2}$ \\
\hline TA (GL) & $=$ & $\begin{array}{l}-1.5 \\
(-0.2)^{* * *}\end{array}$ & + & $\begin{array}{l}1.00 \mathrm{TA} \text { (URSS) } \\
(6.3)^{* * *}\end{array}$ & 0.61 \\
\hline TA (GL) & $=$ & $\begin{array}{l}22.0 \\
(3.3)^{* * * *}\end{array}$ & + & $\begin{array}{l}0.72 \mathrm{TA} \text { (ETR) } \\
(5.5)^{* * * *}\end{array}$ & 0.56 \\
\hline TA (GL) & $=$ & $\begin{array}{l}96.9 \\
(11.7)^{* * *}\end{array}$ & - & $\begin{array}{l}4.74 \mathrm{TCH} \\
(-4.6)^{* * *}\end{array}$ & 0.46 \\
\hline TA (GL) & $=$ & $\begin{array}{l}22.1 \\
(2.6)^{* * *}\end{array}$ & + & $\begin{array}{l}0.98 \mathrm{TA} \text { (ACENTR) } \\
(4.4)^{* * *}\end{array}$ & 0.44 \\
\hline TA (GL) & $=$ & $\begin{array}{l}53.7 \\
(14.5)^{* * *}\end{array}$ & + & $\begin{array}{l}1.47 \text { TINF } \\
(1.8)^{* *}\end{array}$ & 0.11 \\
\hline
\end{tabular}

b) TAUX D'APPROBATION SPECIFIQUE

\begin{tabular}{|c|c|c|c|c|}
\hline $\mathrm{TA}(\mathrm{ECN})$ & $=$ & $\begin{array}{l}89.2 \\
(12.9)^{\text {k***}}\end{array}$ & - & $\begin{array}{l}5.00 \mathrm{TCH} \\
(-5.8)^{* * *}\end{array}$ \\
\hline TA (ECN) & $=$ & $\begin{array}{l}43.5 \\
(12.8)^{* * *}\end{array}$ & + & $\begin{array}{l}1.59 \text { TINF } \\
(2.1)^{* *}\end{array}$ \\
\hline TA (ETR) & $=$ & $\begin{array}{l}-0.3 \\
(-0.1)\end{array}$ & + & $\begin{array}{l}1.38 \mathrm{TA} \text { (ACENTR) } \\
(9.9)^{* * *}\end{array}$ \\
\hline TA (ETR) & $=$ & $\begin{array}{l}-5.4 \\
(-0.5)\end{array}$ & + & $\begin{array}{l}0.95 \text { TA (URSS) } \\
(5.0)^{* * *}\end{array}$ \\
\hline
\end{tabular}

1. La définition des symboles utilisés est la suivante: TA (GL): taux d'approbation global; TA (ECN): taux d'approbation de la politique économique; TA (URSS): taux d'approbation de la politique concernant les relations avec IURSS; TA (ETR): taux d'approbation de la politique étrangère; TCH: taux de chômage; TA (ACENTR): taux d'approbation de la politique en Amérique centrale; TINF: taux d'inflation.

2. Dans le cas de l'analyse de régression simple, nous avons utilisé le $\mathbf{R}^{2}$ comme mesure du pourcentage de variance expliquée puisque le nombre de degrés de liberté était le même (25) d'une équation à l'autre.

(3) Les chiffres entre parenthèses renvoient à la statistique $t$ de Student. Les seuils de signification examinés ont été les suivants pour un test unilatéral: *** $99 \%, * * 95 \%$ et * 90\%. 
Tableau 5: Une analyse de régression multiple de la popularité du président Reagan ${ }^{1}$

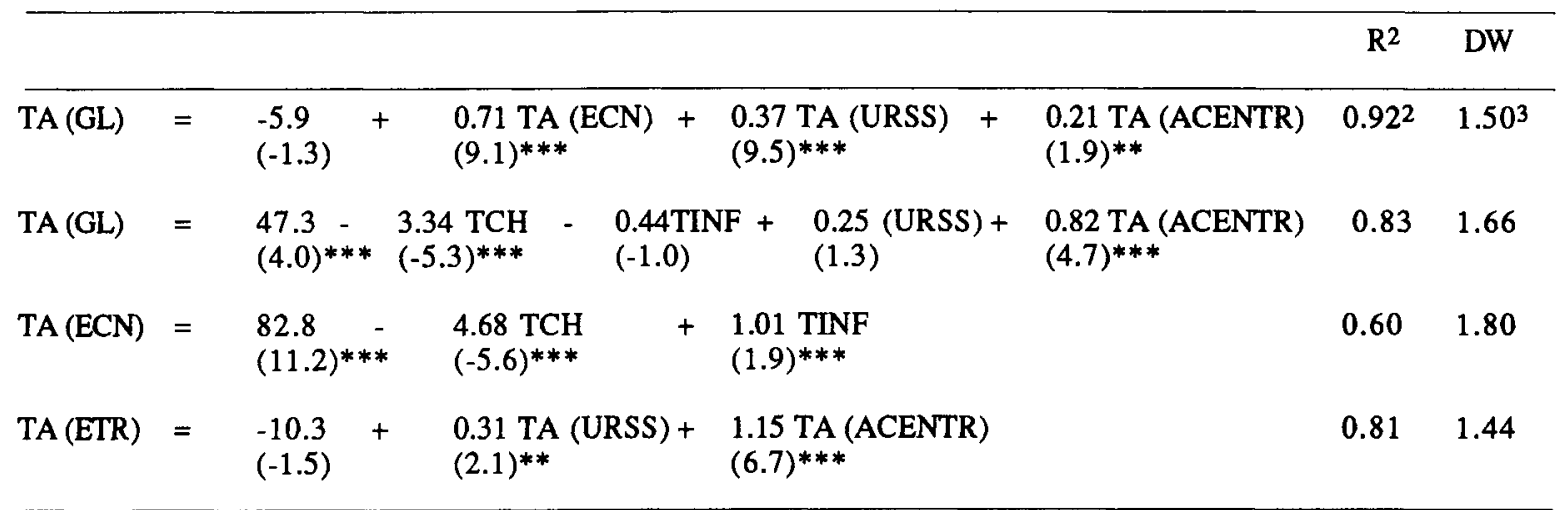

(1) La définition des symboles employés est présentée au tableau précédent.

(2) Puisqu'il s'agit ici d'une analyse de régression multiple, nous avons opté pour le $\mathbf{R}^{2}$ ajusté.

(3) La statistique dite de Durbin-Watson (DW) signale l'absence du problème d'autocorrélation des erreurs résiduelles dans notre analyse de régression (Johnston, 1984). 
Voyons d'abord les résultats concernant les taux d'approbation spécifique avant de revenir au taux d'approbation global. Il convient de noter, d'abord, que l'analyse de régression multiple confirme la prééminence du taux de chômage sur le taux d'inflation, en tant que déterminant de l'évaluation de la politique économique. Là encore, la performance statistique supérieure du taux d'approbation de la politique centre-américaine en tant que déterminant de l'évaluation de la politique étrangère apparaît clairement.

Les résultats antérieurs nous ont amenés à poser que le taux d'approbation de la politique économique pourrait être davantage lié au taux d'approbation global que les taux d'inflation et de chômage, et ce pour deux raisons: 1. l'évaluation de la politique économique peut reposer sur des éléments autres que les taux de chômage et d'inflation; 2 . le lien entre les conditions économiques objectives et l'appréciation de la politique économique emprunte des canaux complexes et encore mal connus, après vingt ans de débats sur ces questions.

Cette idée est confirmée par nos résultats. On notera que l'équation de régression incluant le taux d'approbation de la politique économique affiche une performance statistique nettement supérieure à celle relative aux taux de chômage et d'inflation. Il reste maintenant à expliquer la présence et la performance statistique des deux éléments de politique étrangère de notre équation: les taux d'approbation des politiques soviétique et centre-américaine. La présence de la première variable s'explique aisément, l'impact de la politique des relations avec Moscou sur la popularité ayant été précédemment démontré. Cependant, un problème technique demeure. Tout en contribuant de façon autonome aux fluctuations de la popularité présidentielle, la politique soviétique reste un déterminant important de la politique étrangère (corrélation de 0.71). Or, introduire dans la même équation les relations avec l'URSS et la politique étrangère pose le problème de la collinéarité. Comment alors, introduire ces deux éléments d'évaluation en évitant une telle embûche?

Il faut se rappeler la très forte performance du taux d'approbation de la politique centre-américaine en tant que déterminant du taux d'approbation de la politique étrangère. Ainsi, tout en étant 
fortement corrélée avec ce taux d'approbation (0.89), l'approbation de la politique présidentielle en Amérique centrale l'est moins avec la politique soviétique $(0.60)$ que ne l'est cette dernière avec la politique étrangère. En termes économétriques, le taux d'approbation de la politique centre-américaine joue le rôle d'une variable «proxy». Ce choix s'avère heureux puisque l'équation de régression, incluant le taux d'approbation de la politique economique et les taux d'approbation des politiques soviétique et centre-américaine s'est avérée être la plus performante au plan statistique, tout en permettant de retrouver la hiérarchisation des déterminants précédemment observée: la politique économique, les relations avec Moscou et la politique étrangère en général.

\section{Conclusion}

Le présent texte a étudié les fluctuations de la popularité du président Reagan au cours de ses deux mandats. Il a départagé le poids relatif des deux principaux déterminants de sa popularité que les études antérieures avaient mis au jour: la performance macroéconomique et l'orientation de la politique étrangère. Cette analyse se termine par ces conclusions:

1. Ronald Reagan appartient au groupe de présidents qui, à partir de 1968, n'ont pu compter, en début d'exercice, sur un appui massif de l'électorat. Ces mêmes présidents ont souvent vu ce niveau d'appui, déjà modeste, s'effriter rapidement. Ces deux faits soulignent bien les nuances à apporter lorsque l'on présente Ronald Reagan comme un président populaire. En fait, l'ex-gouverneur de la Californie a joui d'un taux d'approbation très moyen durant l'ensemble de sa présidence et il a été l'un des présidents les moins populaires au cours d'un premier mandat. Tout cela ne doit cependant pas nous faire perdre de vue le fait qu'il a été le seul président de l'après-guerre à briser ce qui avait été, jusque-là, une loi de fer de la politique présidentielle, c'est-à-dire une chute marquée de popularité durant le second mandat. Il faut également noter que la présidence de Ronald Reagan coïncide avec un autre renversement de tendance qui concerne, cette fois, la confiance des 
Américains à l'égard de leurs institutions politiques (Citrin et Green, 1986; Lipset, 1985). Enfin, Reagan n'a pas échappé à cette autre loi de la politique présidentielle qui veut que le parti du président subisse des pertes lors des élections de mi-mandat (Abramovitz et al. 1986).

2. Durant l'ensemble de la présidence Reagan, c'est l'appréciation de la performance de l'économie américaine qui a constitué le facteur explicatif le plus important de sa popularité.

3. L'agenda politique s'est modifié au cours de la période, les préoccupations économiques cédant le pas, en partie, à d'autres préoccupations, en particulier en matière de politique étrangère; il découle de cette évolution que la popularité présidentielle a été davantage liée aux affaires économiques durant le premier mandat et aux affaires étrangères durant le second.

4. L'appréciation de la politique étrangère a été un déterminant de la popularité du président Reagan; cette appréciation a été très liée à l'évaluation de la performance de ce dernier dans les dossiers de l'Amérique centrale et des relations avec l'URSS. Dans ce dernier cas cependant, il semble qu'en plus d'avoir été un déterminant de l'évaluation de la politique étrangère, le dossier des relations avec Moscou ait occupé une place à part et contribué, de façon autonome, aux fluctuations de la popularité présidentielle.

5. L'utilisation du taux d'approbation de la performance économique du président, plutôt que les indicateurs économiques usuels, donne de meilleurs résultats au plan statistique, et suggère que l'impact de l'économie sur la popularité a été sous-estimé jusqu'à maintenant.

Les résultats obtenus montrent bien qu'en dehors des périodes où les Américains sont impliqués directement dans un conflit armé, les déterminants de la popularité de leur président sont: la prospérité économique, la fermeté avec Moscou et, si possible, une politique libérale en ce qui a trait aux autres aspects de la politique étrangère. Une telle hiérarchisation a été profitable aux présidents Eisenhower, Kennedy et Reagan, tandis que son inversion a coûté cher à l'infortuné Jimmy Carter. 


\section{Bibliographie}

ABRAMOVITZ, Alan I. et al., «The President's Party in Midterm Elections: Going from Bad to Worse», American Journal of Political Science, 30, (3), 1986, p. 563-576.

BECK, Nathaniel, «Time-Varying Parameter Regression Models», American Journal of Political Science, 27, (3), 1983, p. 557-600.

CHAPPEL, Henry W. et William R. KEECH, «A New View of Political Accountability for Economic Performance», American Political Science Review, 79, (2), 1985, p. 10-27.

CITRIN, Jack et Donald P. GREEN, «Presidential Leadership and the Resurgence of Trust in Government», British Journal of Political Science, 16, (3), 1986, p. 431-453.

DOWNS, Anthony, An Economic Theory of Democracy, NewYork, Harper \& Row, 1957.

HIBBS, Douglas A., «The Dynamics of Political Support for American Presidents Among Occupational and Partisan Groups», American Journal of Political Science, 26, (1), 1982a), p. 312-333.

HIBBS, Douglas A., «Economic Outcomes and Political Support for American Presidents among Occupational Classes: a Dynamic Analysis», American Political Science Review, 76, (2), 1982b, p. 259-279.

HIBBS, Douglas A., «On the Demand for Economic Outcomes: Macroeconomic Performance and Mass Political Support in the United States, Great Britain and Germany, Journal of Politics, 44, (2), 1982c, p. 426-462.

JOHNSTON, J., Econometric Methods, London, McGraw-Hill, 1984. $\mathrm{KEECH}$, William, «Of Honeymoons and Economic Performance: Comment on Hibbs», American Political Science Review, 76, (2), 1982, p. 280-281.

KENSKI, Henry C., «The Impact of Economic Conditions on Presidential Popularity», Journal of Politics, 39, (3), 1977. p. 764-773.

KERNELL, Samuel, «Explaining Presidential Popularity», American Political Science Review, 72, (2), p. 506-522.

KIEWETT, Roderick D., Macroeconomics and Micropolitics, Chicago, University of Chicago Press, 1982. 
LANOUE, David J., «Economic Prosperity and Presidential Popularity», The Western Political Quarterly, 40, (2), 1987, p. 237-246.

LELOUP, Lance T. et Steven A. SHULL, «Congress Versus the Executive: the «Two Prisidencies» Reconsidered», Social Science Quarterly, 59, (4), 1979, p. 704-719.

LIPSET, Seymour Martin, «Feeling Better: Measuring the Nation's Confidence*, Public Opinion, 8, (1), 1985, p. 6-9.

MACKUEN, Michael B., «Political Drama, Economic Conditions, and the Dynamics of Presidential Popularity", American Journal of Political Science, 27, (2), 1983, p. 165-192.

MUELLER, John E., Wars, Presidents and Public Opinion, New York, John Wiley \& Sons, 1973.

MUELLER, John E., «Presidential Popularity from Truman to Johnson», American Political Science Review, 64, (1), 1970, p. 18-34.

NADEAU, Richard, Modélisation et analyse empirique des fluctuations de court terme du comportement électoral: le cas canadien, Université de Montréal, Département de science politique, thèse de doctorat, chapitre 1, 1988.

NEUSTADT, Richard E. (1960), Presidential Power: the Politics of Leadership, New York, John Wiley \& Sons, chap. 5, 1960.

NORPOTH, Helmuth, «Economics, Politics and the Cycle of Presidential Popularity», Political Behavior, 6, (3), 1984, p. 253-273.

OSTROM, Charles W. et Dennis R. Simon, «Promise and Performance: a Dynamic Model of Presidential Popularity», American Political Science Review, 79, (2), 1985, p. 334-358.

PRESSER, Stanley et Jean M. CONVERSE, «On Stimson's Interpretation of Declines in Presidential Popularity», Public Opinion Quarterly, 40, (4), 1976, p. 538-541.

RIVERS, D et N.L. ROSE, «Passign the President's Program: Public Opinion and the Presidential Influence in Congress», American Journal of Political Science, 29, (2), p. 183-196.

SIGELMAN, Lee et Katlenn SMITH, «Expectation/Disillusion and Presidential Popularity: the Reagan Experience», Public Opinion Quarterly, 49, (1), 1985, p. 209-213.

SIGELMAN, Lee et Katlenn SMITH, «Why Does Presidential Popularity Decline? A Test of the Expectation/Disillusion 
Theory», Public Opinion Quarterly, 47, (2), 1983, p. 310324.

STIMSON, James A., «On Disillusion with the Expectation/ Disillusion Theory: a Rejoinder», Public Opinion Quarterly, 40, (4), 1976a, p. 541-543.

STIMSON, James A., «Public Support for American Presidents: a Cyclical Model», Public Opinion Quarterly, 40, (1), 1976b, p. 1-21.

WHITELEY, Paul, «Inflation, Unemployment, Government Popularity - Dynamic Models for the United States, Britain and West Germany», Electoral Studies, 3, (1), 1984, p. 324.

WHITELEY, Paul, «Politico-Econometric Estimation in Britain: an Altemative Interpretation», in Paul Whiteley ed., Models of Political Economy, London, Sage Publications, 1980, p. 8599.

WIDALVSKY, A., Perspectives on the Presidency, Boston, Little Brown, 1975. 